\title{
Fusion-fission of superheavy compound nuclei produced in reactions with heavy ions beyond $\mathrm{Ca}$
}

\author{
M.G. Itkis ${ }^{1}$, F. Goennenwein ${ }^{2}$, I.M. Itkis ${ }^{1}$, G.N. Knyazheva ${ }^{1}$, E.M. Kozulin ${ }^{1}$, A.A. Bogachev ${ }^{1}$, and E. Vardaci ${ }^{3}$ \\ ${ }^{1}$ FLNR, Joint Institute of Nuclear Research, 141980 Dubna, Russia \\ ${ }^{2}$ Physikalihes Institute, Universitaet Tuebingen, 72076 Tuebingen, Germany \\ ${ }^{3}$ INFN and Dipartamento di Scienze Fisiche dell'Universita di Napoli, Napoli, Italy
}

\begin{abstract}
Total Kinetic Energy - Mass distributions of fission-like fragments for the reactions of ${ }^{22} \mathrm{Ne},{ }^{26} \mathrm{Mg},{ }^{36} \mathrm{~S},{ }^{48} \mathrm{Ca},{ }^{58} \mathrm{Fe}$ and ${ }^{64} \mathrm{Ni}$ ions with actinides leading to the formation of superheavy compound systems with $\mathrm{Z}=108-120$ at energies near the Coulomb barrier have been measured. Fusion-fission cross sections were estimated from the analysis of mass and total kinetic energy distributions. It was found that the fusion probability drops by three orders of magnitude for the formation of the compound nucleus with $Z=120$ obtained in the reaction ${ }^{64} \mathrm{Ni}+{ }^{238} \mathrm{U}$ compared to the formation of the compound nucleus with $Z=112$ obtained in the reaction ${ }^{48} \mathrm{Ca}+{ }^{238} \mathrm{U}$ at the excitation energy of the compound nucleus of about $45 \mathrm{MeV}$. From our analysis it turns out that the reaction ${ }^{64} \mathrm{Ni}+{ }^{238} \mathrm{U}$ is not suitable for the synthesis of element $\mathrm{Z}=120$.
\end{abstract}

\section{Introduction}

A large success was achieved in the synthesis of superheavy elements (SHE) with $\mathrm{Z}=108-118$ in the reactions of "cold" and "warm" fusion. Reactions with $\mathrm{Pb} / \mathrm{Bi}$ targets, leading to lowly excited compound nucleus $(\mathrm{CN})$, are used for "cold" fusion [1], whereas the reactions with actinide targets, leading to excited $\mathrm{CN}$, are used for "warm" fusion [2].

In the "cold" fusion reactions the excitation energy of $\mathrm{CN}$ is $10-20 \mathrm{MeV}$ near the reaction threshold, the $\mathrm{CN}$ formation cross section is suppressed strongly by competing quasifission (QF) $[3,4]$ and deep-inelastic reactions. The balance between the fusion and $\mathrm{QF}$ strongly depends on the entrance channel properties, such as mass-asymmetry, deformation of interacting nuclei, collision energy and the Coulomb factor $Z_{1} Z_{2}$. These processes take place in the "warm" fusion reactions also, but their contributions into the reaction cross sections are smaller compared with the "cold" reactions due to the difference in the entrance channel.

The excitation energy of a formed $\mathrm{CN}$ is about 30$40 \mathrm{MeV}$ in "warm" fusion reactions and the de-excitation of $\mathrm{CN}$ to the ground state is due to the emission of three or four neutrons and several $\gamma$-rays. Nevertheless in the "warm" fusion reactions the formed $\mathrm{CN}$ is more neutron rich and nearer to the closed neutron shell at $\mathrm{N}=184$ than in the case of "cold" fusion reactions. Consequently, the "warm" fusion reactions are more preferable for synthesis of the SHE. At FLNR double magic ${ }^{48} \mathrm{Ca}$ ions were used for the productions of elements with $\mathrm{Z}=112-118$ [2]. The cross sections of SHE in these reactions do not change practically with increasing atomic number of $\mathrm{CN}$ and maintain the level of a few picobarn.

Nuclei with $Z>118$ cannot be synthesized in ${ }^{48} \mathrm{Ca}$ induced reactions since ${ }^{249} \mathrm{Cf}$ is the heaviest target material available for these purposes. One of the possible ways for further progress in the field of the SHE synthesis is to use the complete fusion reactions of ${ }^{238} \mathrm{U}$, ${ }^{244} \mathrm{Pu}$ and ${ }^{248} \mathrm{Cm}$ nuclei with heavier projectiles, such as ${ }^{58} \mathrm{Fe}$ or ${ }^{64} \mathrm{Ni}$, leading to the formation of even heavier elements with $Z=120-124$ and $N=179-183$. However, the increase of projectile charge leads to the increasing of the QF contribution.

This paper presents the results of the experimental investigations of the properties of binary reaction products obtained in the reactions of ${ }^{22} \mathrm{Ne},{ }^{26} \mathrm{Mg},{ }^{36} \mathrm{~S}$, ${ }^{48} \mathrm{Ca},{ }^{58} \mathrm{Fe}$ and ${ }^{64} \mathrm{Ni}$ with actinides at energies near the Coulomb barrier. Binary reaction products were measured by using the two-arm time-of-flight spectrometer CORSET [5].

Previously in the experimental investigations the symmetric fragment region with mass $\mathrm{A}_{\mathrm{CN}} / 2 \pm 20 \mathrm{u}$ was often attributed with $\mathrm{CN}$-fission. However, a realistic description of deep inelastic scattering, $\mathrm{QF}$ and $\mathrm{CN}$ fission processes in low energy heavy ion collisions shows [6] that the potential energy surface for these systems is strongly modulated by shell effects and leads to the appearance of deep valleys corresponding to the formation of well bound magic nuclei. In accordance with these calculations, at least three paths leading to the formation of fissionlike fragments can be distinguished: asymmetric QF caused by the influence of proton shells with $\mathrm{Z}=28,82$ and neutron shells with $\mathrm{N}=50,126$; symmetric $Q F$ determined by the shells with $Z=50$ and $\mathrm{N}=82$; $\mathrm{CN}$-fission leading to the formation of symmetric fragments. Therefore, in present analysis we supposed that the mass region $A_{C N} / 2 \pm 20 u$ is populated by the $C N$ fission fragments, as well as the QF fragments and paid a special attention to the properties of symmetric fragments in order to assign probabilities of $\mathrm{CN}$-fission and $\mathrm{QF}$ processes. 


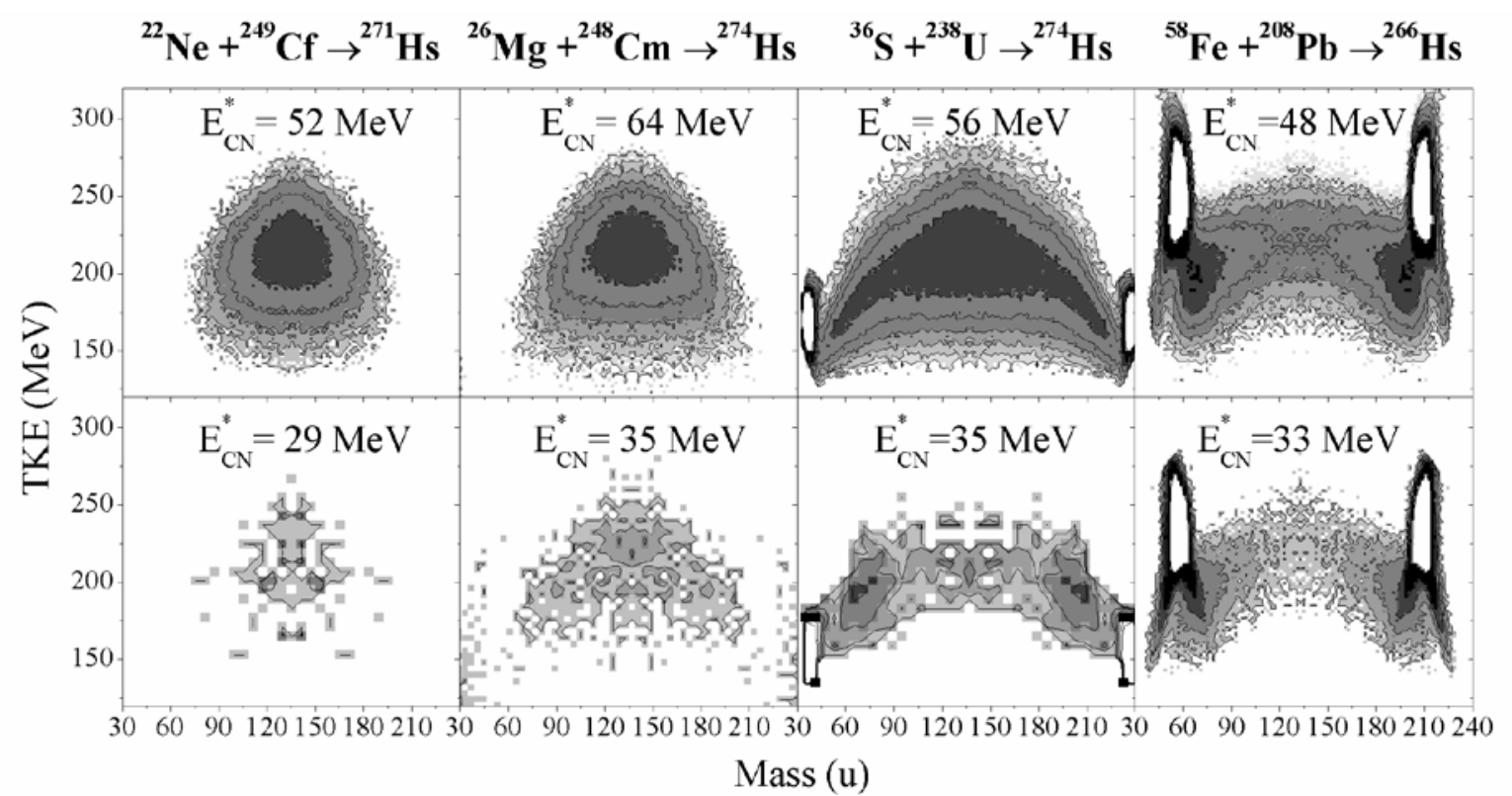

Fig. 1. Mass-energy distributions of binary reaction fragments for the reactions ${ }^{22} \mathrm{Ne}+{ }^{249} \mathrm{Cf},{ }^{26} \mathrm{Mg}+{ }^{248} \mathrm{Cm},{ }^{36} \mathrm{~S}+{ }^{238} \mathrm{U},{ }^{58} \mathrm{Fe}+{ }^{208} \mathrm{~Pb}$ leading to the formation of elements with $Z=108$ at energies above (top panel) and below (bottom panel) the Coulomb barrier.

\section{$2 \mathrm{CN}$-fission and QF in the reactions leading to the Hs composite systems}

Mass and Total Kinetic Energy (TKE) distributions of binary reaction products obtained in the reactions ${ }^{22} \mathrm{Ne}+{ }^{249} \mathrm{Cf},{ }^{26} \mathrm{Mg}+{ }^{248} \mathrm{Cm},{ }^{36} \mathrm{~S}+{ }^{238} \mathrm{U},{ }^{58} \mathrm{Fe}+{ }^{208} \mathrm{~Pb}$ have been measured. All reactions lead to Hs isotopes.

In the chosen reactions the parameter of massasymmetry in the entrance channel $\alpha$ varies strongly: for the reaction ${ }^{58} \mathrm{Fe}+{ }^{208} \mathrm{~Pb}: \alpha=0.571$, for ${ }^{36} \mathrm{~S}+{ }^{238} \mathrm{U}: \alpha=$ 0.737, for ${ }^{26} \mathrm{Mg}+{ }^{248} \mathrm{Cm}: \alpha=0.810$, and for ${ }^{22} \mathrm{Ne}+{ }^{249} \mathrm{Cf}: \alpha$ $=0.838$. As demonstrated in Fig. 1 the TKE-mass distributions change with decreasing asymmetry $\alpha$ in the entrance channel from symmetric for incoming ${ }^{22} \mathrm{Ne}$-ions to strongly asymmetric for incoming ${ }^{58} \mathrm{Fe}$-ions. These changes are understood as reflecting the relative contributions of $\mathrm{CN}$-fission and $\mathrm{QF}$ to the fission process excited $\mathrm{Hs}^{*}$ isotopes as depending on the reaction studied.

It is clearly seen that even at similar $\mathrm{CN}$ excitation energies the TKE-mass distributions are vastly different for these reactions. In the case of the reactions ${ }^{22} \mathrm{Ne}+{ }^{249} \mathrm{Cf}$ and ${ }^{26} \mathrm{Mg}+{ }^{248} \mathrm{Cm}$ the mass distributions have a near Gaussian shape with no evidence for asymmetric fission. The reactions are considered to be mainly attributed to the $\mathrm{CN}$-fission process. For the ${ }^{36} \mathrm{~S}+{ }^{238} \mathrm{U}$ reaction the mass distributions of the fission-like fragments change markedly. This difference in mass distribution for the ${ }^{26} \mathrm{Mg}+{ }^{248} \mathrm{Cm}$ and ${ }^{36} \mathrm{~S}+{ }^{238} \mathrm{U}$ reactions is connected with an increasing contribution of the QF process for the ${ }^{36} \mathrm{~S}$-induced reaction. At low excitation energies $\mathrm{QF}$ is the dominant process for the reaction ${ }^{36} \mathrm{~S}+{ }^{238} \mathrm{U}$. At higher excitation energies the mass distribution becomes symmetric and similar to the reaction ${ }^{26} \mathrm{Mg}+{ }^{248} \mathrm{Cm}$ though - due to a remaining trace of QF - slightly wider.

The TKE-mass distribution for the ${ }^{58} \mathrm{Fe}+{ }^{208} \mathrm{~Pb}$ reaction has a wide two-humped shape even at excitation energy of $48 \mathrm{MeV}$ (well above the Bass barrier). For this reaction the QF process dominates at energies below and above the Bass barrier. This reaction takes a special place due to the fact that one of the partners is double-magic lead. It is known that for heavy-ion induced reaction the formation of QF fragments is determined by the strong influence of the nuclear shell at $\mathrm{Z}=82$ and $\mathrm{N}=126$ (double magic lead). Therefore, for this reaction the $\mathrm{QF}$ fragments overlap strongly with quasi-elastic and deep-inelastic events. It is clearly seen on the TKE-mass matrices in the Fig. 1.

For the reactions ${ }^{26} \mathrm{Mg}+{ }^{248} \mathrm{Cm}$ and ${ }^{36} \mathrm{~S}+{ }^{238} \mathrm{U}$ the cross section of the evaporation residues (ER) amounts to a few nanobarns $[7,8]$ and contributes insignificantly to the fusion cross section. Thus we can estimate the fusion probability using the measured TKE-mass distributions as the ratio between the number of events attributed to fusion-fission in the frame of the Liquid Drop Model and all fission-like fragments. The so-obtained fusion probabilities as a function of the energy above the barrier are presented as open symbols in Fig. 2 for these reactions. As it follows from the present experimental data, the properties of the entrance channel strongly affect the reaction dynamics. At the excitation energy near the barrier the estimated values of $\mathrm{P}_{\mathrm{CN}}$ are about $70 \%$ in the case of the $\mathrm{Mg}$-induced reaction and about $25 \%$ in the $\mathrm{S}$-induced reaction. The enhancement of subbarrier fusion for the reaction ${ }^{26} \mathrm{Mg}+{ }^{248} \mathrm{Cm}$ was observed in experiment by measuring the ERs [7], caused by the orientation and coupled-channel effects on fusion with deformed targets. In the case of the ${ }^{36} \mathrm{~S}+{ }^{238} \mathrm{U}$ reaction the sub-barrier fusion enhancement was not observed, although such an effect could be expected owing to the deformation of ${ }^{238} \mathrm{U}$. The measured ER cross sections in the reaction ${ }^{36} \mathrm{~S}+{ }^{238} \mathrm{U}$ are lower than those of the ${ }^{26} \mathrm{Mg}+{ }^{248} \mathrm{Cm}$ reaction. The reduction of the ER cross section for the reaction ${ }^{36} \mathrm{~S}+{ }^{238} \mathrm{U}$ may be explained by the suppression of fusion due to the dominance of the QF process as compared to the more asymmetric ${ }^{26} \mathrm{Mg}+{ }^{248} \mathrm{Cm}$ reaction. Thus, as it follows from the ER 
cross section measurements, the fusion probability depends mainly on the asymmetry of the projectile-target combination. This conclusion agrees with the results of the present work.

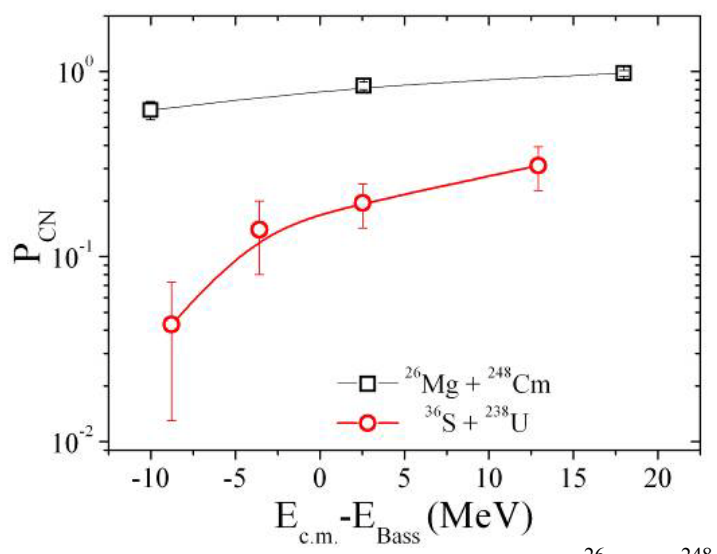

Fig. 2. The fusion probabilities in the reactions ${ }^{26} \mathrm{Mg}+{ }^{248} \mathrm{Cm}$ and ${ }^{36} \mathrm{~S}+{ }^{238} \mathrm{U}$.

\section{Reactions with $\mathrm{Ca}, \mathrm{Fe}$ and $\mathrm{Ni}$ ions}

Unfortunately, the transition to heavier projectile results in the large increase in the Coulomb factor $\mathrm{Z}_{1} \mathrm{Z}_{2}$ that is crucial for the competition between $\mathrm{CN}$-fission and QF. The TKE-mass distributions for the reactions ${ }^{58} \mathrm{Fe}+{ }^{244} \mathrm{Pu}$ and ${ }^{64} \mathrm{Ni}+{ }^{238} \mathrm{U}$ (leading to the formation of the same composite system with $\mathrm{Z}=120$ and $\mathrm{N}=182$ ) at the $\mathrm{CN}$ excitation energies of about $45 \mathrm{MeV}$ are presented in Fig. 3. At first sight the distributions are similar for both reactions, consisting of a wide two-humped shape with a large QF component peaked around the mass $215 \mathrm{u}$. In the formation of the QF component the closed shell at $\mathrm{N}=50$ seems to be effective together with the shell $\mathrm{Z}=82$ and $\mathrm{N}=126$ and leads to the shift of the QF peak from mass $208 \mathrm{u}$, observed in the reaction ${ }^{48} \mathrm{Ca}+{ }^{238} \mathrm{U}$, to $215 \mathrm{u}$ in the case of the ${ }^{58} \mathrm{Fe}+{ }^{244} \mathrm{Pu}$ and ${ }^{64} \mathrm{Ni}+{ }^{238} \mathrm{U}$ reactions.

However, at the same $\mathrm{CN}$ excitation energy the mass drift to the symmetry (estimated as the distance between masses corresponding to the maximum and half maximum of QF yields) is 22 nucleons in the case of the ${ }^{58} \mathrm{Fe}$ reaction and only 11 nucleons in the case of the ${ }^{64} \mathrm{Ni}$ ions. It is significant that the mass drift to the symmetry is about 34 nucleons for the ${ }^{48} \mathrm{Ca}+{ }^{238} \mathrm{U}$ at the same $\mathrm{CN}$ excitation energy. The contribution of the symmetric fragments with masses $\mathrm{A}_{\mathrm{CN}} / 2 \pm 20 \mathrm{u}$ to all fission-like events is about $8 \%$ and $4 \%$ for $\mathrm{Fe}$ and $\mathrm{Ni}$-ions, respectively. The average TKE's are similar for asymmetric QF fragments for the both reactions, while in the symmetric mass region the TKE for the $\mathrm{Fe}+\mathrm{Pu}$ reaction is higher than for the $\mathrm{Ni}+\mathrm{U}$. The solid line corresponds to the parabolic dependence following from the Liquid Drop Model and the Viola systematics for the TKE. The dispersions of the TKE are different for the two reactions: it is bigger in the case of Fe-ion induced reaction. In the reaction ${ }^{64} \mathrm{Ni}+{ }^{238} \mathrm{U}$ the dispersion does practically not depend on the fragment mass and its mean value is about $350 \mathrm{MeV}^{2}$, while for the reaction ${ }^{58} \mathrm{Fe}+{ }^{244} \mathrm{Pu}$ the dispersion increases for symmetric fragments and its mean value is about $610 \mathrm{MeV}^{2}$.
According to the existing experimental data on the dispersion of the TKE distribution of $\mathrm{CN}$-fission of heavy and superheavy nuclei [10] the dispersion does practically not change for $\mathrm{CN}$ with $\mathrm{Z}^{2} / \mathrm{A}^{1 / 3}$ up to $\sim 1000$ and increases linearly for heavier $\mathrm{CN}$. The extrapolation of this experimental dependence gives the value of about $550 \mathrm{MeV}^{2}$ for the fission of ${ }^{302} 120$. Hence, for the reaction with $\mathrm{Ni}$-ions the TKE dispersion is lower than this value, whereas it is higher in the case of Fe-ions.

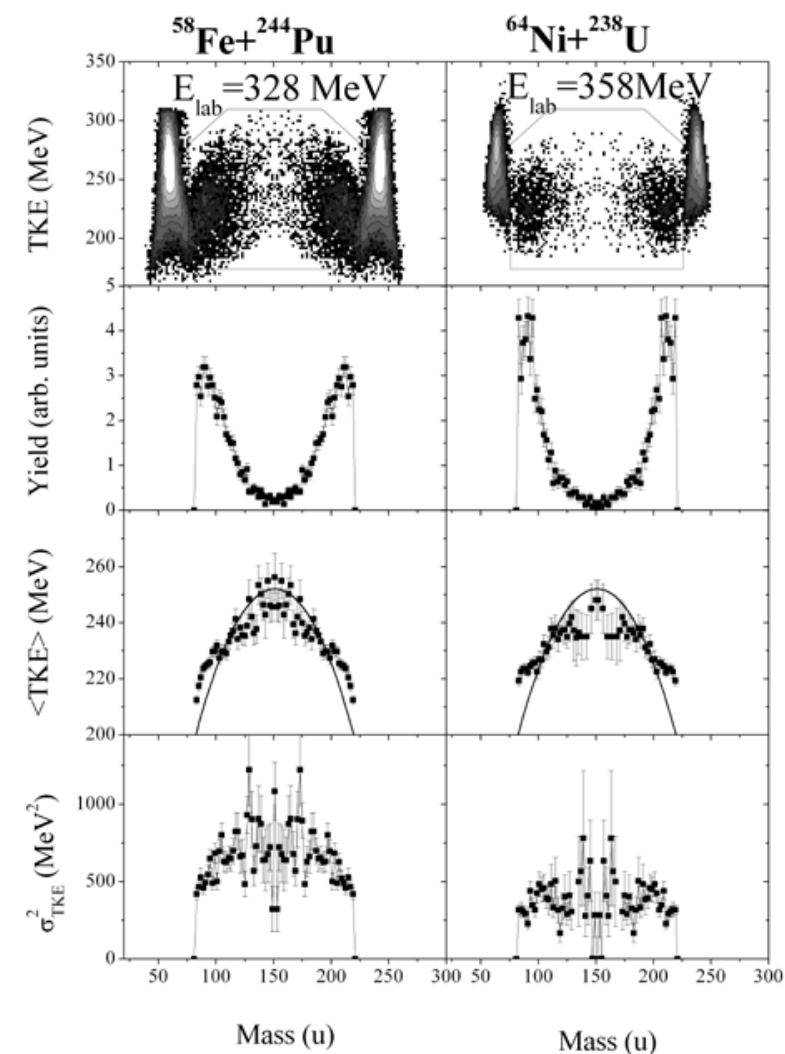

Fig. 3 . From top to bottom: the two-dimensional TKE-M matrices, yields, average TKEs and TKE dispersions of fragments inside the contour lines on TKE-M matrices (bottom panels) in the ${ }^{58} \mathrm{Fe}+{ }^{244} \mathrm{Pu}$ and ${ }^{64} \mathrm{Ni}+{ }^{238} \mathrm{U}$ reactions at the $\mathrm{CN}$ excitation energies of about $45 \mathrm{MeV}$.

In figure 4 the TKE distributions of fission-like fragments in the mass region $\mathrm{A}_{\mathrm{CN}} / 2 \pm 20 \mathrm{u}$ for the reactions ${ }^{48} \mathrm{Ca}+{ }^{238} \mathrm{U},{ }^{58} \mathrm{Fe}+{ }^{244} \mathrm{Pu}$ and ${ }^{64} \mathrm{Ni}+{ }^{238} \mathrm{U}$ are presented. It is seen that the TKE distributions have a complex structure for all reactions.

In fact, it is known that in the $\mathrm{CN}$-fission process the average TKE of the partner fragments is substantially independent on the excitation energy and shows a typical Gaussian-like shape. The TKE distributions were described by the sum of the three Gaussians for all the reactions studied.

From the Viola systematics [9] we infer that the average TKE is in a first approximation a linear function of the Coulomb barrier $\mathrm{Z}^{2} / \mathrm{A}^{1 / 3}$ whereas from the systematics in Ref. [10] we can estimate the variance of the TKE distribution. For the ${ }^{286} 112 \mathrm{CN}$ formed in the reaction ${ }^{48} \mathrm{Ca}+{ }^{238} \mathrm{U}$, the variance of the TKE distribution is about $400 \mathrm{MeV}^{2}$ and the TKE is $226 \mathrm{MeV}$. Given the considerable good agreement with the systematics, we associate the middle component of the TKE distribution with the $\mathrm{CN}$-fission process. Since the asymmetric 
fragments have lower TKE's than the symmetric ones, the low energy component of the experimental TKE distribution may be associated with fragments originating from the asymmetric QF process.

The high energy part may arise instead from the symmetric mode of the QF. Furthermore, we note that the mean TKE of this mode is about $40 \mathrm{MeV}$ higher that the mean TKE for the $\mathrm{CN}$-fission process. Considering that both processes give rise to symmetric mass fragments, the difference in mean TKE can be taken as an evidence that in the QF process a complete dissipation of the entrance channel energy does not occur. As a consequence, the symmetric fragments with high TKE do not originate from complete fusion because the final fragments retain part of the entrance channel total kinetic energy.

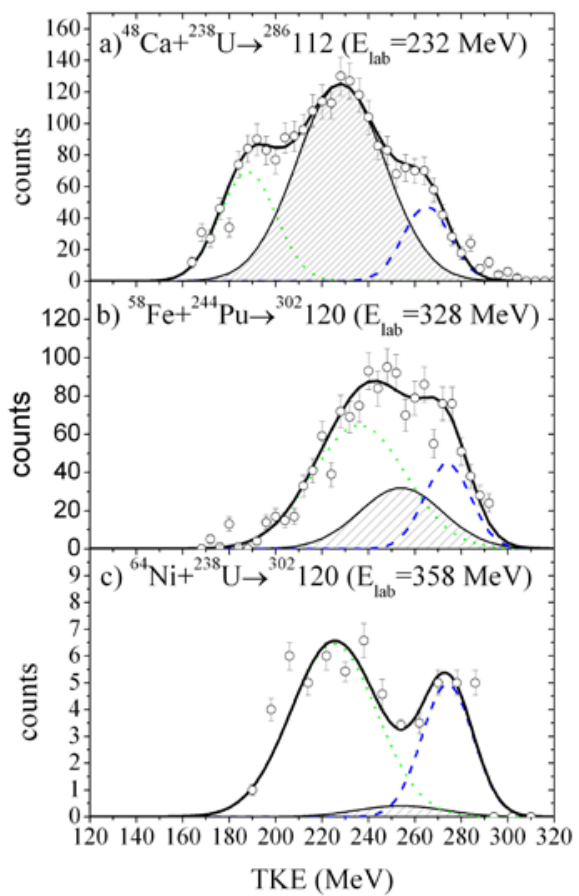

Fig. 4. TKE distributions of fragments with masses $A_{C N} / 2 \pm 20$ $\mathrm{u}$ for the reaction ${ }^{48} \mathrm{Ca}+{ }^{238} \mathrm{U},{ }^{58} \mathrm{Fe}+{ }^{244} \mathrm{Pu}$ and ${ }^{64} \mathrm{Ni}+{ }^{238} \mathrm{U}$. The hatched region corresponds to $\mathrm{CN}$-fission with energy taken from the Viola systematics.

In contrast to ${ }^{48} \mathrm{Ca}+{ }^{238} \mathrm{U}$, for the reaction ${ }^{64} \mathrm{Ni}+{ }^{238} \mathrm{U}$ the TKE distribution has more pronounced low and high energy components (see fig. 4c), while the component with average value of $252 \mathrm{MeV}$ (corresponding to the Viola systematics) is highly hindered. Because of the low statistics, only an upper value for the relative yield of the $\mathrm{CN}$-fission component can be reasonably given. Table 1 gives the relative contribution of all symmetric fragments in the mass range $\mathrm{A}_{\mathrm{CN}} / 2 \pm 20 \mathrm{u}$ and symmetric fragments with TKE corresponding to the Viola systematics.

The obtained capture cross sections are about a few hundred millibarns for $\mathrm{Ca}$ and $\mathrm{Ni}$ induced reactions, whereas the formation of symmetric fragments is one order of magnitude less for the reaction ${ }^{64} \mathrm{Ni}+{ }^{238} \mathrm{U}$. Yet, in the case of the $\mathrm{Ca}+\mathrm{U}$ at the energies above the barrier, approximately $70 \%$ of the events have the TKE expected for the $\mathrm{CN}$-fission process, whereas in the case of the $\mathrm{Ni}$ reaction only a few percents of the symmetric fragments have the TKE compatible with the Viola prediction for the ${ }^{302} 120 \mathrm{CN}$-fission. While the ${ }^{64} \mathrm{Ni}+{ }^{238} \mathrm{U}$ reaction has lower excitation energy at the reaction threshold energy, the $\mathrm{CN}$-fission cross section is suppressed by stronger QF process.

Table 1. The relative contributions of $\mathrm{CN}$-fission to the capture cross section $\left(\sigma_{\text {cap }}\right)$ at $\mathrm{CN}$ excitation energy of around $45 \mathrm{MeV}$.

\begin{tabular}{|c|c|c|c|}
\hline Reaction & $\sigma_{\mathrm{ACN} / 2 \pm 20} / \sigma_{\text {cap }}$ & $\sigma_{\mathrm{CNF}} / \sigma_{\mathrm{ACN} / 2 \pm 20}$ & $\sigma_{\mathrm{CNF}} / \sigma_{\text {cap }}$ \\
\hline${ }^{48} \mathrm{Ca}+{ }^{238} \mathrm{U}$ & $(12 \pm 2) \%$ & $(68 \pm 3) \%$ & $(8 \pm 4) \%$ \\
\hline${ }^{58} \mathrm{Fe}+{ }^{244} \mathrm{Pu}$ & $(8 \pm 3) \%$ & $\leq 25 \%$ & $\leq 2 \%$ \\
\hline${ }^{64} \mathrm{Ni}+{ }^{238} \mathrm{U}$ & $(4 \pm 1) \%$ & $\leq 5 \%$ & $\leq 0.2 \%$ \\
\hline
\end{tabular}

\section{Conclusions}

Previously to distinguish between $\mathrm{QF}$ and $\mathrm{CN}$ fission the angular and mass distributions of fissionlike fragments were used. The present analysis of the TKE distributions of fragments with masses $\mathrm{A}_{\mathrm{CN}} / 2 \pm 20 \mathrm{u}$ for different reactions studied shows that the variance of the TKE distribution is sensitive to the presence of the QF process. At an excitation of $\mathrm{CN}$ larger than $40 \mathrm{MeV}$ (when the shell in $\mathrm{CN}$-fission is practically disappeared) the broadening of the TKE distribution points out to the presence of the both $\mathrm{QF}$ and $\mathrm{CN}$-fission processes. The narrowing of the TKE distributions indicates that $\mathrm{QF}$ is a dominant process.

The fusion probabilities for different reactions were estimated from the simultaneous analysis of the TKE and mass distributions. It was found that the relative contribution of QF to the capture cross section increases rapidly with the increasing Coulomb reaction factor $Z_{1} Z_{2}$.

A further progress in the field of synthesis of SHE can be achieved using hot fusion reactions between actinide nuclei and ${ }^{48} \mathrm{Ca}$ ions as well as ${ }^{50} \mathrm{Ti},{ }^{54} \mathrm{Cr},{ }^{58} \mathrm{Fe}$ ions. At the transition from $\mathrm{Ca}$ to $\mathrm{Ni}$ projectiles the contribution of the QF process rises sharply.

\section{References}

1. S. Hofmann and G. Münzenberg, Rev. Mod. Phys. 72, 733 (2000).

2 . Yu. Ts. Oganessian, J. Phys. G: Nucl. Part. Phys. 34, R165-R242 (2007).

3. R. Bock et al., Nucl. Phys. A388, 334 (1982).

4. M.G. Itkis et al., Inter. Jour. Mod. Phys. E 16, 957 (2007).

5. E.M. Kozulin et al., Instr. Exp. Tech. 51, 44 (2008).

6 . V.I. Zagrebaev and W. Greiner, J. Phys. G 31, 825 (2005)

7. J. Dvorak et al., Phys. Rev. Lett. 100, 132503 (2008).

8. R. Graeger et al., Phys. Rev. C 81, 061601(R) (2010).

9. V.E. Viola, Nucl. Data Tables A1 391 (1966).

10. M. G. Itkis and A.Ya. Rusanov, Phys. Part. Nucl. 29, 160 (1998). 